\title{
Right ventricular and epicardial tumors from benign metastasizing uterine leiomyoma
}

\author{
Matthew Williams, DO, Tomas Salerno, MD, and Anthony L. Panos, MD, Miami, Fla
}

\footnotetext{
From the Division of Cardiothoracic Surgery, University of Miami Miller School of Medicine and Jackson Memorial Hospital, Miami, Fla.

Disclosures: Authors have nothing to disclose with regard to commercial support.

Received for publication Aug 10, 2015; revisions received Sept 2, 2015; accepted for publication Sept 14, 2015; available ahead of print Nov 11, 2015.

Address for reprints: Anthony L. Panos, MD, University of Miami/Jackson Memorial Hospital, 1611 NW 12th Ave, Holtz 3072 (R-114), Miami, FL 33136 (E-mail: apanos@med.miami.edu).

J Thorac Cardiovasc Surg 2016;151:e21-4

$0022-5223 / \$ 36.00$

Copyright (C) 2016 by The American Association for Thoracic Surgery

http://dx.doi.org/10.1016/j.jtcvs.2015.09.059
}

Primary intracardiac tumors are rare and found in less than $0.1 \%$ of the population. ${ }^{1}$ Secondary cardiac tumors are more common and found in $1 \%$ of the population at postmortem. $^{2}$ They can be found in the epicardium, myocardium, or endocardium. Uterine leiomyomas more commonly have intravenous extension along the wall of the inferior vena cava to the heart. ${ }^{3-9}$

\section{CASE SUMMARY}

A 51-year-old woman with a history of a $19 \times 17-\mathrm{cm}$ uterine fibroid presented to the University of Miami Miller School of Medicine and Jackson Memorial Hospital with worsening abdominal pain (Figure 1). She was scheduled for exploratory laparotomy, hysterectomy, and bilateral salpingo-oophorectomy. A systolic cardiac murmur was

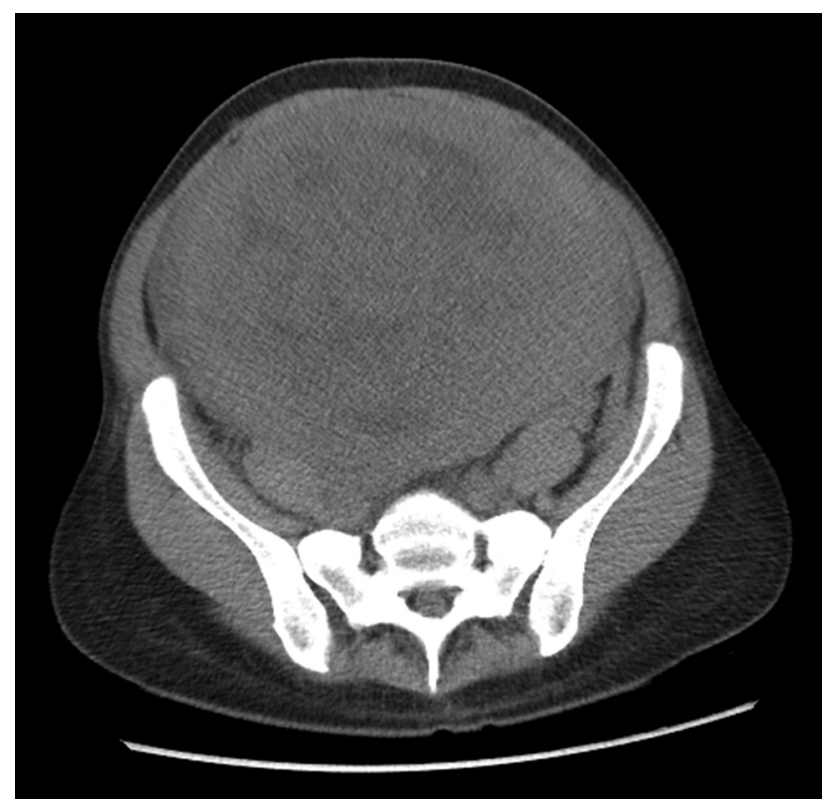

FIGURE 1. Left: A $19 \times 17-\mathrm{cm}$ uterine fibroid on computed tomography scan.

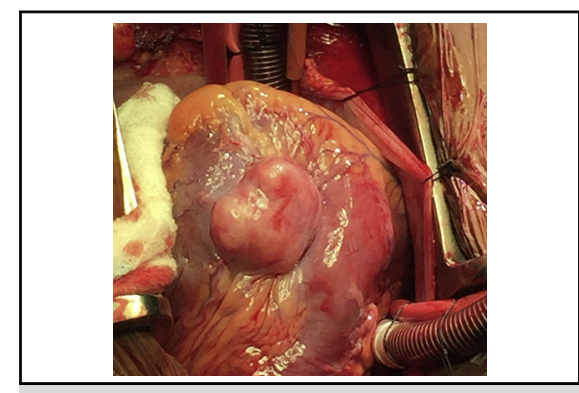

RV tumor and epicardial tumor involving the left anterior descending coronary artery.

\section{Central Message}

We report the first known case report of a benign uterine leiomyoma with an epicardial tumor. The coronary artery was also involved.

See Editorial Commentary page e 25 .

heard during the preoperative workup, and a transthoracic echocardiogram was obtained. A $4.5 \times 2.5-\mathrm{cm}$ ball valve mass was found in the right ventricle intermittently obstructing the pulmonary artery (Figure 2). Cardiac magnetic resonance imaging was obtained that showed the right ventricular (RV) mass and an epicardial mass (Figures 3-5). A computed tomography scan of the chest showed 6 subcentimeter bilateral pulmonary nodules.

Surgery was performed via median sternotomy, aortic arterial cannulation, and bicaval venous cannulation. After

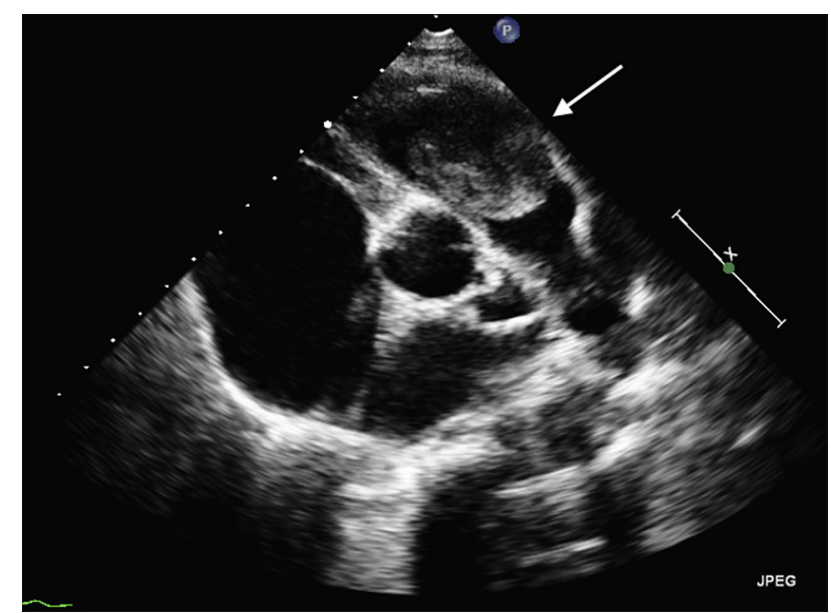

FIGURE 2. Right: Short-axis aortic valve transthoracic echocardiogram view showing $4.5-\mathrm{cm} \mathrm{RV}$ outflow tract mass. 


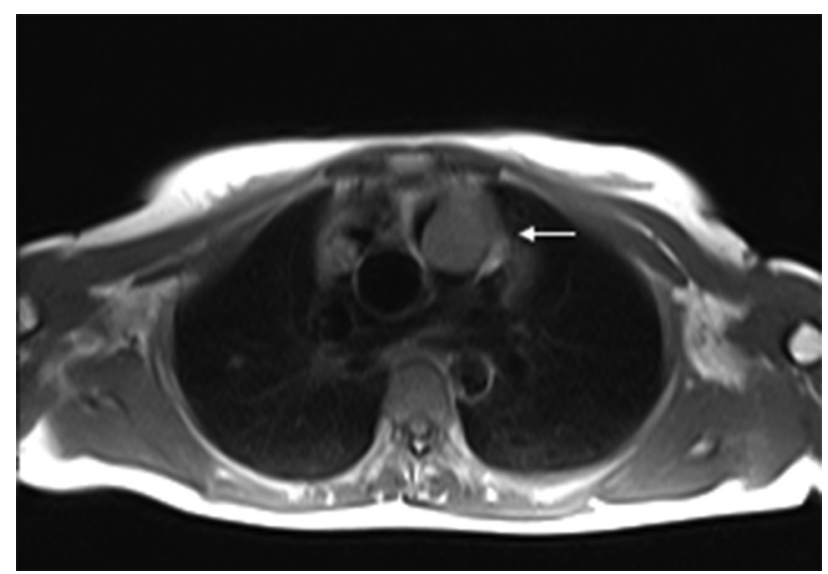

FIGURE 3. Top left: Cardiac magnetic resonance imaging showing RV mass.

placing the patient on total cardiopulmonary bypass, a right atriotomy was performed, the anterior leaflet of the tricuspid valve was retracted, and the RV mass was identified and brought into the field. The pedunculated tumor was attached to the right side of the interventricular septum and was transected at its base (Figure 6). A Tru-Cut (CareFusion, San Diego, Calif) biopsy was taken of the epicardial mass. The left anterior descending coronary artery was interrupted by the mass and could be seen running on the mass's right lateral side (Figure 7). The 2 masses were not structurally related. The patient had an ejection fraction of more than $55 \%$ on transesophageal echocardiogram with no ventricular wall dysfunction. The right atriotomy was closed in a running fashion with 4-0 Prolene suture, and the patient was weaned from cardiopulmonary bypass.

Tissue pathology of the RV tumor supported the diagnosis of a benign leiomyoma. Histologically, the tumor was composed of spindle-shaped cells with less than 1 mitosis per 10 high-power fields, no cellular atypia, and no necrosis. Immunohistochemical staining was positive

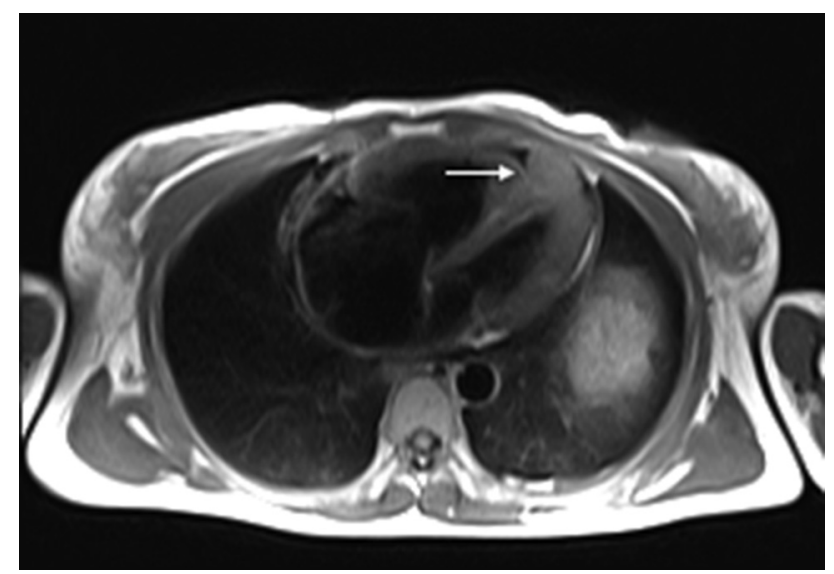

FIGURE 4. Top right: Cardiac magnetic resonance imaging showing epicardial mass.

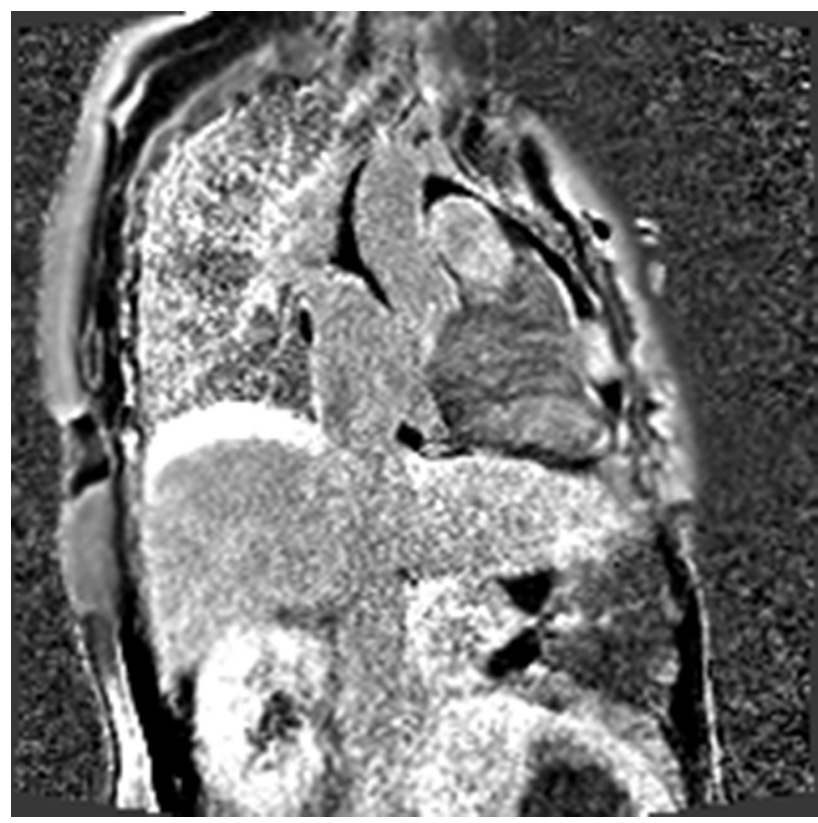

FIGURE 5. Bottom: Magnetic resonance imaging showing the relationship of the 2 masses.

for desmin, estrogen receptors, and progesterone receptors. Ki-67 was less than $10 \%$, representing a low proliferation rate and benign disease process (Figure 8).

\section{DISCUSSION}

Leiomyomas represent less than $1 \%$ of primary cardiac tumors, but they are the most common benign uterine tumor. ${ }^{1,2}$ To our knowledge, 3 case reports of primary cardiac leiomyomas exist. ${ }^{10-12}$ Cardiac metastases may present in

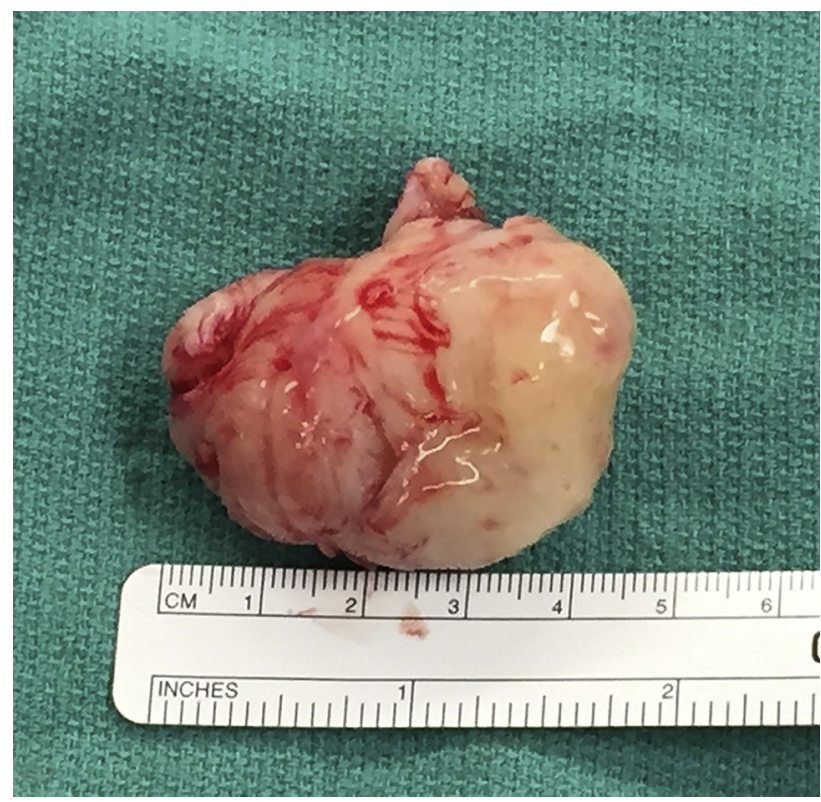

FIGURE 6. Left: Excised RV tumor. 


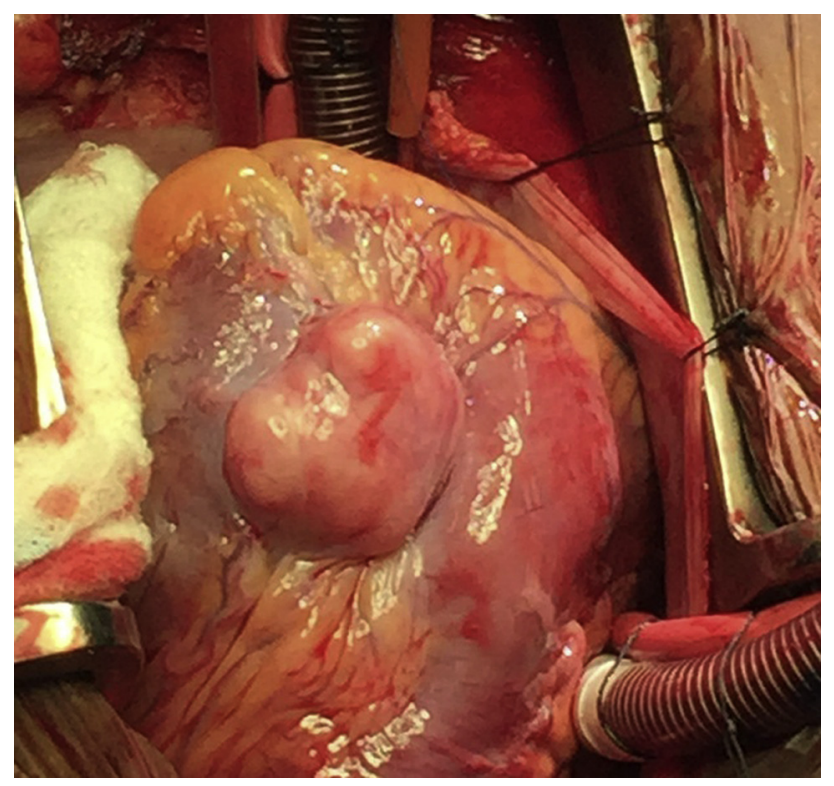

FIGURE 7. Right: Epicardial tumor. The left anterior descending coronary artery is interrupted by the mass, and the left anterior descending coronary artery runs on the right lateral border of the mass.

synchrony with the primary tumor or years later. ${ }^{6}$ More commonly, uterine leiomyomas have intravenous spread that extends to the heart. ${ }^{3-9}$ Pulmonary metastasis occurred with cardiac metastasis in $80 \%$ of the reported cases. ${ }^{13-16}$ Distant metastases also can occur to the lymph nodes, brain, bone, skin, eye, and spinal cord. This is the first case report of a metastatic epicardial leiomyoma. The tumor displaced the left anterior descending coronary artery, but did not cause compromised coronary blood flow and was left in situ.

Curative resection would have been possible if this patient had isolated cardiac metastases or resectable pulmonary metastases. In a case series of 6 patients with uterine leiomyomas and metastases to the heart, the only patient with a recurrence had an incomplete cardiac tumor resection at the time of surgery. ${ }^{3}$ Another case report documented a 16-year disease-free survival after resection of a benign metastasizing uterine leiomyoma (BMUL) solitary pulmonary nodule. ${ }^{17}$ Our patient had diffuse bilateral pulmonary nodules.

A standardized treatment for BMUL with hormonal therapy has not been established. Pulmonary metastases are present in the majority of BMULs. ${ }^{18}$ BMULs are hormonally dependent, and the majority are estrogen receptor and progesterone receptor positive. Regression of metastatic lesions has been seen after a decrease in estrogen levels, such as after surgical oophorectomy or during postpartum or postmenopausal states, although recurrent progression of
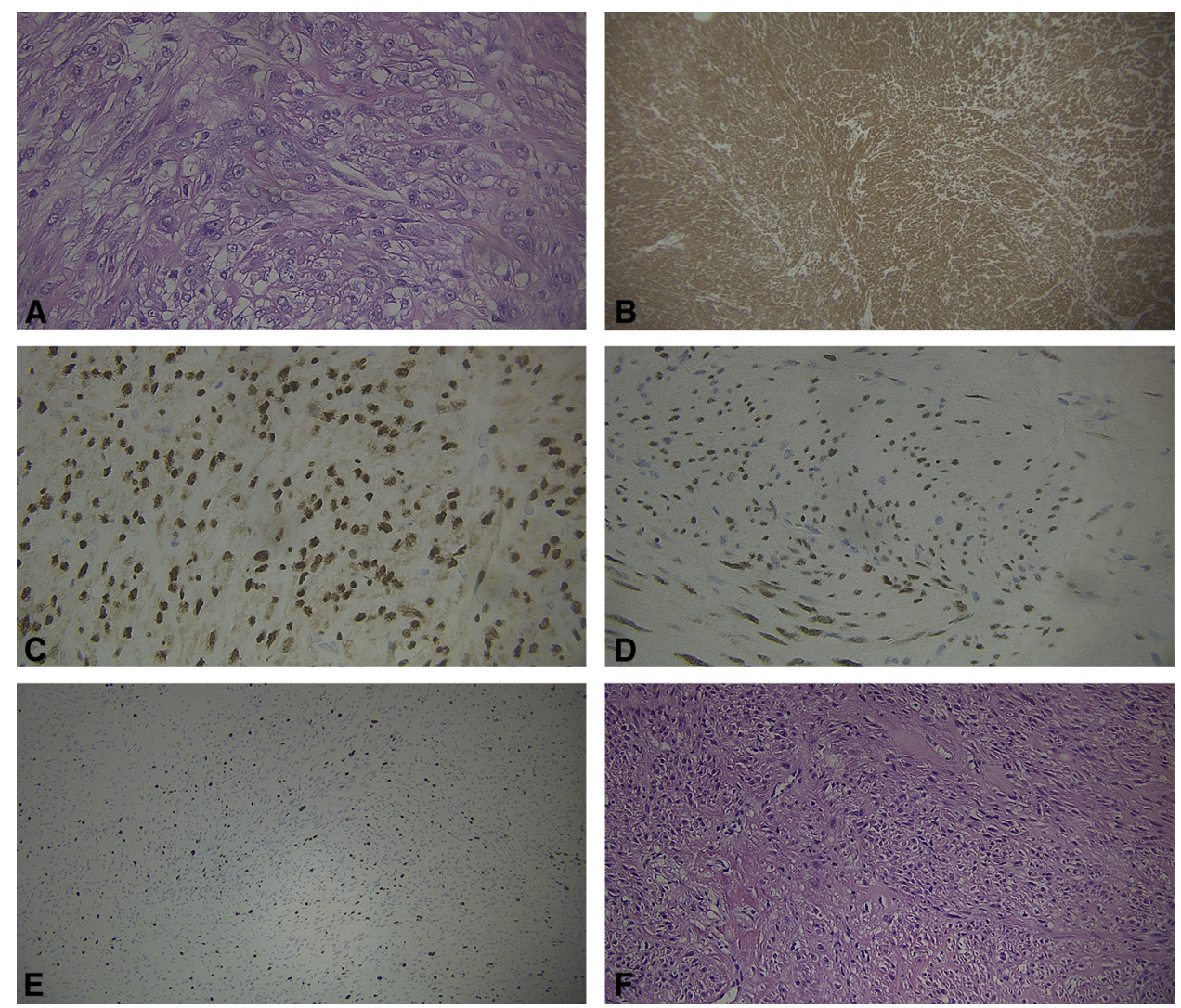

FIGURE 8. A, (top left) Hematoxylin-eosin stain (high power). B, Desmin stain (low power). C, Progesterone receptor stain (high power). D, Estrogen receptor stain (high power). E, Ki-67 stain. F, Epicardial tumor, hematoxylin-eosin stain (mid power). 
tumor a few years postpartum was seen in 1 patient. ${ }^{19}$ A perimenopausal woman in a case series had no progression of diffuse pulmonary nodules at the 2-year follow-up without hormonal therapy. ${ }^{17}$

There are mixed reports regarding the response of BMUL to hormonal therapy, such as gonadotropin-releasing hormone $(\mathrm{GnRH})$, raloxifene, anastrozole, or luteinizing hormone-releasing hormone. One case report of a premenopausal woman with BMUL showed a stable size of the pulmonary nodules after 3 years of treatment with GnRH. ${ }^{19}$ A case report of a patient with diffuse metastatic BMUL pulmonary nodules and a symptomatic pleural effusion had resolution of the pleural effusion but only mild radiology improvement of the pulmonary nodules with a course of raloxifene and anastrozole.$^{18}$ Hormonal therapy, including luteinizing hormone-releasing hormone, has been shown to treat symptoms such as menstrual bleeding and pain from bone or pulmonary metastases, but tumor regression was absent. ${ }^{20-23}$

On the basis of a review of the literature, observation of pulmonary nodules in low estrogen and progesterone states, such as in postmenopausal or perimenopausal women, is safe. Hormonal therapy should be considered for premenopausal women. The response to hormonal therapy varies from lack of progression to regression of the tumor.

\section{CONCLUSIONS}

Our patient was started on letrozole, an aromatase inhibitor, 1 month after surgery because of her perimenopausal symptoms of hot flashes. It was continued for 1 month and then was switched to Lupron Depot (AbbVie, North Chicago, Ill), a GnRH agonist. At her 2-month follow-up, she denied signs of clinical worsening. A hysterectomy and repeat computed tomography scan of the chest have been recommended at 3 months from the time of her cardiac surgery. Although the epicardial tumor and pulmonary nodules were left behind, our palliative procedure is likely to benefit this patient because of the reported benign nature of the residual disease.

\section{References}

1. Reynen K. Frequency of primary tumors of the heart. Am J Cardiol. 1996;77:107.

2. Lam K, Dickens P. Tumors of the heart. A 20 year experience with review of 12485 consecutive autopsies. Arch Pathol Lab Med. 1993;117:1027-31.

3. Anselmi A, Tsiopoulos V. Case series of resection of pelvic leiomyoma extending into the right heart: surgical safeguards and clinical follow-up. J Cardiovasc Med. 2010;11:583-6.

4. Liu B, Liu C. Intravenous leiomyomatosis with inferior vena cava and heart extension. J Vasc Surg. 2009;50:897-902.

5. Gaudina M, Spatuzza P. Surgical management of a uterine leiomyoma extending through the inferior vena cava into the right heart. Heart Vessels. 2002;17: $80-2$.

6. Vural C, Ozen O. Intravenous lipoleiomyomatosis of the uterus with cardiac extension: a case report. Pathol Res Pract. 2011;207:131-4.

7. Tierney W, Ehrlich C. Intravenous leiomyomatosis of the uterus with extension into the heart. Am J Med. 1980;69:471-5.

8. Katsumata T, Shinfeld A. Pelvic leiomyoma in the right atrium. Ann Thorac Surg. 1998;66:2095-6.

9. Kolaczyk K, Chamier K. Pulmonary benign metastasizing leiomyoma from the uterine leiomyoma: a case report. Pol J Radiol. 2015;80:107-10.

10. Qin C, Chen L. Giant primary leiomyoma of the right ventricle. J Card Surg. 2010;25:169-71.

11. Song L, Wang. Primary leiomyoma: a rare space occupying lesion in the right ventricle. Ann Thorac Surg. 2014;97:324-6.

12. Melo IS, Belo F. Primary cardiac leiomyoma of the ventricular septum: a rare form of pediatric intracardiac tumor. Pediatr Cardiol. 2012;33:649-51.

13. Takemura G, Takatsu Y. Metastasizing uterine leiomyoma. A case with cardiac and pulmonary metastasis. Pathol Res Pract. 1996;192:622-9.

14. Thukkani N, Ravichandran PS. Leiomyomatosis metastatic to the tricuspid valve complicated by pelvic hemorrhage. Ann Thorac Surg. 2005;79:707-9.

15. Galvin S, Wademan B. Benign metastasizing leiomyoma: a rare metastatic lesion in the right ventricle. Ann Thorac Surg. 2010;89:279-81.

16. Consamus E, Reardon M. Metastasizing leiomyoma to heart. Methodist Debakey Cardiovasc J. 2014;10:251-4.

17. Ki E, Hwang S. Benign metastasizing leiomyoma of the lung. World J Surg Oncol. 2013;11:279.

18. Rivera J, Christopoulos S. Hormonal manipulation of benign metastasizing leiomyomas: report of two cases and review of the literature. J Clin Endocrinol Metab. 2004;89:3183-8.

19. Egberts J, Schafmayer C. Benign abdominal and pulmonary metastasizing leiomyoma of the uterus. Arch Gynecol Obstet. 2006;274:319-22.

20. Friedman A, Barbieri R. A randomized, double-blind trial of a gonadotropinreleasing hormone agonist (leuprolide) with or without medroxyprogesterone acetate in the treatment of leiomyomata uteri. Fertil Steril. 1988;49:404.

21. Carr B, Marshburn P. An evaluation of the effect of gonadotropin-releasing hormone analogs and medroxyprogesterone acetate on uterine leiomyomata volume by magnetic resonance imaging: a prospective, randomized, double blind, placebo-controlled, crossover trial. J Clin Endocrinol Metab. 1993;76:1217.

22. Maheux R, Samson Y. Utilization of luteinizing hormone releasing hormone agonist in pulmonary leiomyomatosis. Fertil Steril. 1987;48:315-8.

23. Nakanishi S, Nakano K. So-called benign metastasizing leiomyoma of the lung presenting with bone metastasis. Nihon Kokyukin Gakkai Zasshi. 1999;37:146-50. 